\title{
Boosting the Efficiency: Unconventional Waveform Design for Efficient Wireless Power Transfer
}

\author{
Alírio Boaventura, Student Member, IEEE, Daniel Belo, Student Member, IEEE, Ricardo Fernandes, \\ Student Member, IEEE, Ana Collado, Senior Member, IEEE, Apostolos Georgiadis, Senior Member, \\ IEEE, and Nuno Borges Carvalho, Senior Member, IEEE
}

\begin{abstract}
Traditionally, wireless power is delivered through single carrier continuous wave $(\mathrm{CW})$ signals. Most research efforts to enhance the efficiency of wireless power transfer systems has been confined to the circuit level design. However, in the recent years, attention has been paid to the waveform design for wireless power transmission. It has been found that signals featuring high PAPR can provide efficiency improvement when compared to $\mathrm{CW}$ signals. A number of different approaches have been proposed such as multisines/multi-carrier (OFDM) signals, chaotic signals, harmonic signals, UWB signals, intermittent $\mathrm{CW}$ or white noise. This paper reviews these techniques with focus on the multisines/multi-carrier, harmonic signal and chaotic signals. Theoretical explanation for the efficiency improvement is provided accompanied by experimental results. Circuit design considerations are presented for the receiver side, and efficient transmission architectures are also described, with emphasis on spatial power combining.

Index Terms - Wireless Power Transfer, Non-constant Envelope Signals, Multi-sines, Chaotic, harmonic signals, UWB, OFDM, Spatial Power Combining, Rectifier, diode.
\end{abstract}

\section{INTRODUCTION}

$\mathrm{P}$ ower transfer without wires has been carried out for many years using single carrier continuous wave $(\mathrm{CW})$ signals. However, it has been demonstrated recently that proper waveform design (e.g. featuring high peak-toaverage power ratio - PAPR) can improve the efficiency of wireless energy transfer, especially for low power levels. Curiously, the first electromagnetic wave successfully generated, transmitted and detected by Heinrich Hertz in 1887 was in fact a non-constant envelope signal, consisting of damped waves [1]. This type of signal was used for many years for signal transmission (in wireless telegraphy) and also in some wireless power transfer experiments

This work was supported by the Portuguese Foundation for Science and Technology (FCT) under Doctoral Scholarship SFRH/BD/80615/2011 and Project CREATION EXCL/EEI-TEL/0067/2012, and by the Spanish Ministry of Economy and Competitiveness and FEDER funds through the project TEC2012-39143, the Generalitat de Catalunya under grant 2014 SGR 1551 and the COST Action IC1301 Wireless Power Transmission for Sustainable Electronics (WIPE).

Alirio Boaventura, Daniel Belo, Ricardo Fernandes and Nuno Borges Carvalho are with the Instituto de Telecomunicações, Departmento de Electrónica, Telecomunicações e Informatica, Universidade de Aveiro, Aveiro 3819-193, Portugal (e-mail: a34422@ua.pt; nbcarvalho@ua.pt).

Ana Collado and Apostolos Georgiadis are with Centre Tecnologic de Telecomunicacions de Catalunya (CTTC), Castelldefels 08860, Spain (email: acollado@cttc.es; ageorgiadis@cttc.es).

Color versions of one or more of the figures in this paper are available online at http://ieeexplore.ieee.org. performed by Nikola Tesla in 1894 and following years [2]. Nevertheless, damped waves, which presents a large spectrum occupancy, were later replaced by CW signals, first generated in 1902 by Ernst Alexanderson's high frequency alternators [3]. Continuous wave signals have been the choice for WPT applications since WPT experiments were recovered by W. Brown in the 60's (some decades after the suspension of Tesla experiments), until the present days.

Currently, WPT technology is of interest in scientific but also in industry areas. Energy transfer efficiency is a central issue in WPT, and ultimately imposes system coverage range, performance and reliability. At the receiver side, the efficiency optimization of the RF-DC converter circuits is a must and is traditionally accomplished through improved circuit design [4-8].

Alternatively, the RF-DC conversion efficiency can be boosted by selecting a proper excitation signal [9-16]. For instance, the use of high PAPR multi-sine signals has proved to increase the efficiency of RF-DC circuits when compared to CW signals $[8,11]$.

This paper reviews a number of approaches that use unconventional waveforms for WPT. This includes intermittent $\mathrm{CW}$ (ICW), UWB, chaotic signals, multi-sine and harmonic signals, with focus on the last three categories. Theoretical explanation for the efficiency improvement is provided accompanied by experimental results.

\section{TRADITIONAL WIRELESS POWER TRANSFER USING CW SIGNALS}

Typically, the transfer of energy wirelessly is realized by using a $\mathrm{CW}$ signal. Figure 1 depicts the block diagram of a WPT system. At the transmitter side, DC energy is converted into a CW RF signal, which is radiated through the transmitting antenna. The RF energy is collected by the receiving antenna and is forwarded to the RF-DC converter that converts it back to DC energy to power up electronic circuits. 


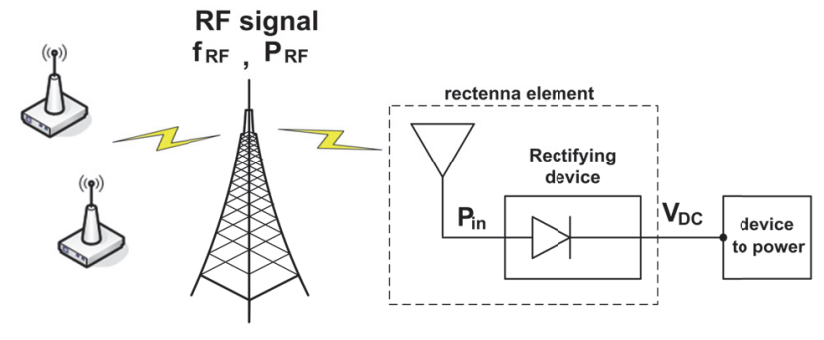

Fig. 1 Simplified schematic of a wireless power transmission (WPT) system.

\section{A. $\quad C W R F-D C$ Conversion}

Figure 2 depicts basic topologies of RF-DC converters or rectifier circuits. The first two topologies (shunt diode and series diode) are envelope detectors, which ideally provide an output DC voltage proportional to the input power (due to their predominant second order behavior). The third configuration is a voltage multiplier or charge pump (in this case a 2-level one), which is used to boost the output voltage amplitude. Single diode topologies are in principle more power efficient than charge pumps (see Fig.3) and are often used in energy harvesting circuits. On the other hand, charge pumps are capable of providing higher voltage levels and are commonly utilized in circuits that need voltage excursion rather than power efficiency, such as passive RFID tags.

The rectification of a $\mathrm{CW}$ signal using a single diode rectifier is illustrated in Fig.4. Considering the exponential transfer function characteristic of a rectifying diode (I-V curve), a CW voltage signal at the input yields an output current signal with a certain DC component. On the other hand, if a high PAPR signal (e.g. a multi-sine signal) is applied to the same device, a higher output DC component, even considering the same average power. This is illustrated in Fig.5.

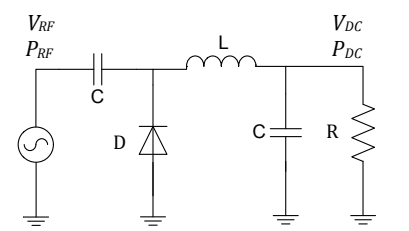

(a)

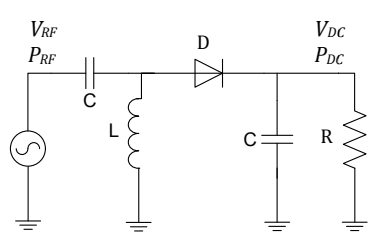

(b)

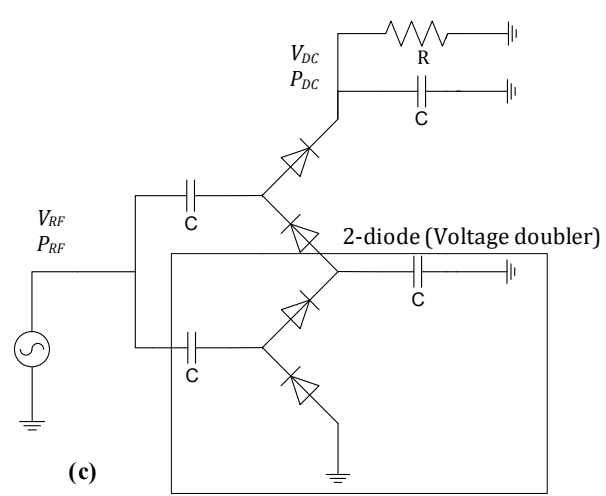

Fig. 2 Basic RF-DC converter topologies, a) single shunt diode, b) single series diode, c) voltage multiplier (2-level).
Typical efficiency curves of different RF-DC converter circuits are presented in Fig.3. The RF-DC power efficiency is defined as the ratio between the output DC power (Pout) and the input RF power (Pin). From Fig.3, two conclusions can be drawn. First, the efficiency depends on the selected circuit topology, and the lower the number of devices, the higher the efficiency. This is explained because of the need for a minimum amount of input power to switch on the rectifying devices.

Second, the efficiency depends on the available power at the input of the circuit: for low power levels the efficiency is low because the rectifying device is not completely switched on. As the input power increases the efficiency increases and reaches a maximum value right before the input amplitude reaches the diode breakdown voltage. After this point, diode reverse current starts to be significant and the efficiency drops. This power dependency imposes the necessity of optimizing the design for the expected input power level.

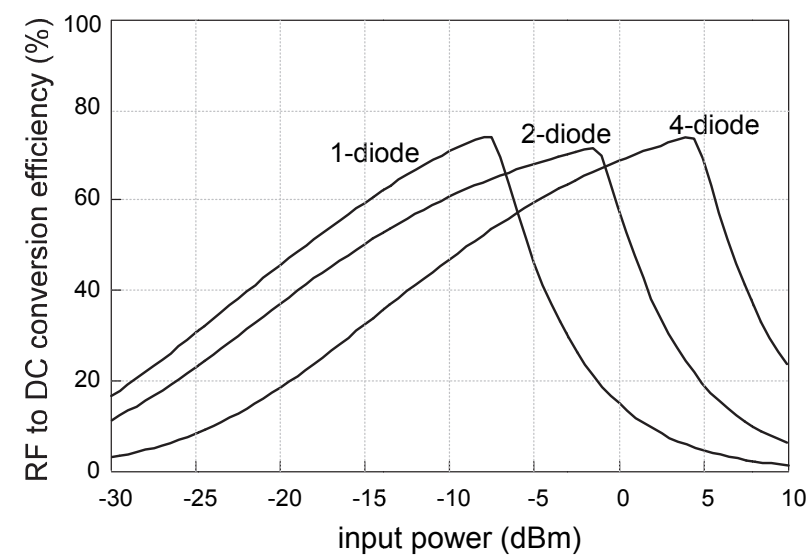

Fig. 3 RF to DC conversion efficiency versus RF input power level for different rectifier topologies. efficiency versus RF input power.

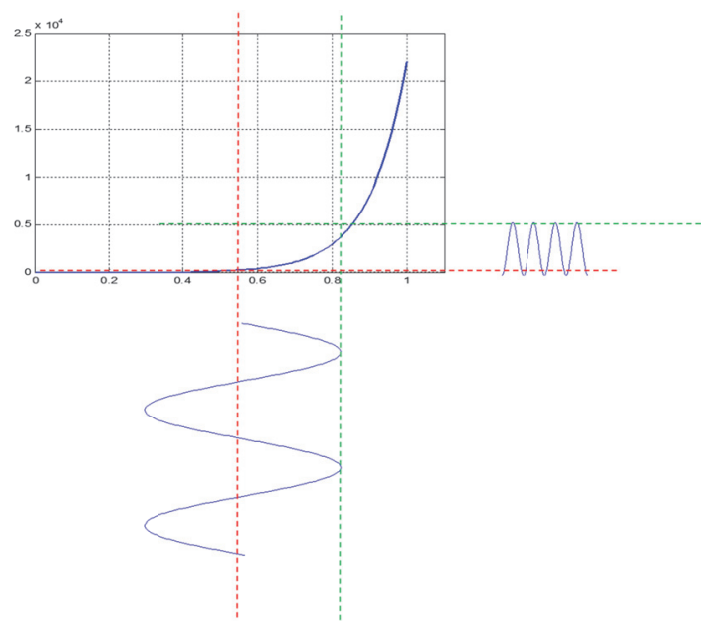

Fig. 4 Rectification of a sinusoidal signal.

If the rectifying device is approximated by a fourth order even order polynomial series [13], when a sinusoidal signal 
is used as input (1), the output DC component (after filtering) is given by (2).

$$
x(t)=B \cos \left(\omega_{1} t+\varphi_{1}\right)
$$

$y_{D C}=\frac{B^{2} k_{2}}{2}+\frac{3 B^{4} k_{4}}{8}$

This simple model considers only the first two even order coefficients $\left(k_{2}\right.$ and $\left.k_{4}\right)$ of the Taylor expansion. Assuming that the second order is dominant, the DC component can be approximated to be proportional to the square of the input signal amplitude, that is, proportional to the average input power.

\section{UNCONVENTIONAL WAVEFORMS FOR WPT}

In order to illustrate the rectification process of a high PAPR signal and to show the mechanism by which the efficiency is improved over single-carrier case, let us consider a high PAPR multi-sine signal (Fig.5) as described in [13]. If such signal is applied to the input of a rectifying circuit, the output DC component will be higher compared to a $\mathrm{CW}$, even using the same average power. This is due to the higher voltage peaks exhibited by the multi-sine waveform, which are able to overcome the diode threshold barrier more efficiently than the (average) powerequivalent $\mathrm{CW}$ signal. This can be seen in Fig.5. Equation (3) is a four-tone evenly spaced multi-sine signal, whose tones have amplitude $\mathrm{A}$, phases $\varphi_{1}, \varphi_{2}, \varphi_{3}$ and $\varphi_{4}$ and frequencies $\omega_{1}, \omega_{2}=\omega_{1}+\Delta \omega, \omega_{3}=\omega_{1}+2 \Delta \omega, \omega_{4}=\omega_{1}+3 \Delta \omega$, respectively, where $\Delta \omega$ is the frequency spacing between the tones. After filtering out the RF and baseband components, the DC output of a rectifier as modelled in [13] is given by equation (4).

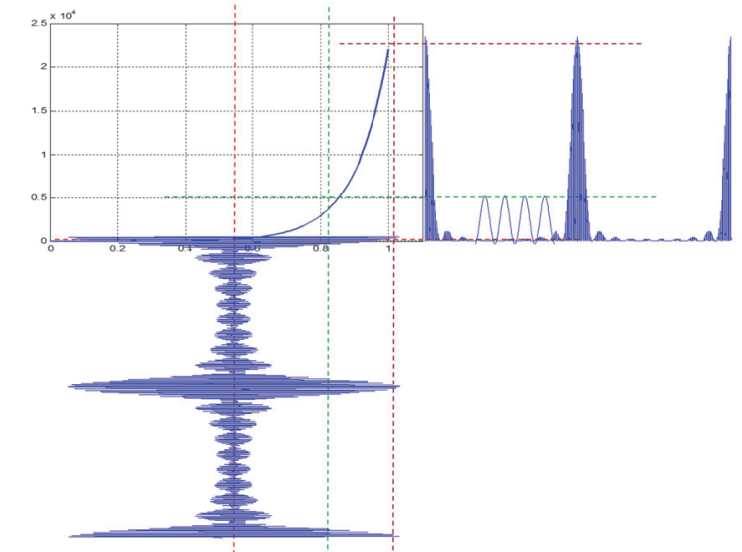

Fig. 5 Rectification of a high PAPR multi-sine signal.

$$
\begin{aligned}
& x(t)=A \cos \left(\omega_{1} t+\varphi_{1}\right)+A \cos \left(\omega_{2} t+\varphi_{2}\right)+ \\
& +A \cos \left(\omega_{3} t+\varphi_{3}\right)+A \cos \left(\omega_{4} t+\varphi_{4}\right)
\end{aligned}
$$

$$
\begin{aligned}
& y_{D C}=\frac{4 A^{2} k_{2}}{2}+\frac{21 A^{4} k_{4}}{2}+\frac{3 A^{4} k_{4}}{2} \cos \left(2 \varphi_{3}-\varphi_{2}-\varphi_{4}\right) \\
& +\frac{3 A^{4} k_{4}}{2} \cos \left(-2 \varphi_{2}+\varphi_{1}+\varphi_{3}\right)+3 A^{4} k_{4} \cos \left(\varphi_{1}-\varphi_{2}-\varphi_{3}+\varphi_{4}\right)
\end{aligned}
$$

By equation (4) it can be concluded that the output is a function of the input phases, and it can be optimized by choosing the adequate phase arrangement that maximizes the signal PAPR. Maximum PAPR value occurs when all the tones are aligned with $0^{\circ}$ phase $\left(\varphi_{1}=\varphi_{2}=\varphi_{3}=\varphi_{4}=\right.$ $0^{\circ}$ ) or when the phase progression between adjacent tones is constant $\left(\phi_{i+1}-\phi_{i}=\Delta \phi\right)$ [13-17]. Equally-spaced tone frequencies $\left(\omega_{i}=\omega_{0}+i \cdot \Delta \omega\right)$ is also a necessary condition. By increasing the PAPR of the multi-sine, the output DC voltage also increases due to the fact the PAPR peaks will charge the output capacitor to a higher value. However, a proper signal and circuit design is required to accommodate increased voltage ripple imposed by the low frequency component of the multi-sine envelope.

As multi-sine signals, a number of other signals that present high PAPR characteristic can excite rectifying devices more efficiently than $\mathrm{CW}$ signals, and will be studied in the next sections. These include, for instance, pulsed or intermittent $\mathrm{CW}$, chaotic, white noise, multi-carrier OFDM, UWB and harmonic signals.

In order to evaluate the improvements obtained with the unconventional schemes, compared to the $\mathrm{CW}$, a FOM (figure of merit) is defined, namely the $R F-D C$ efficiency gain $\left(G_{\eta}\right)$, which relates the DC power collected from a single carrier with the DC power obtained with an unconventional waveform with the same average power $\left(P_{R F(C W)}=P_{R F(U)}\right)$.

$$
\begin{aligned}
& G_{\eta}(d B)=10 \cdot \log 10\left(\frac{\eta_{(U)}}{\eta_{(C W)}}\right)= \\
& 10 \cdot \log 10\left(\frac{P_{D C(U)} / P_{R F(U)}}{P_{D C(C W)} / P_{R F(C W)}}\right)= \\
& =10 \cdot \log 10\left(\frac{P_{D C(U)}}{P_{D C(C W)}}\right)=10 \cdot \log 10\left(\frac{V_{D C(U)}^{2}}{V_{D C(C W)}^{2}}\right)
\end{aligned}
$$

where, $P_{D C(C W)}$ refers to the output DC power obtained in a rectifier circuit when using a single carrier signal at its input, and $P_{D C(U)}$ refers to the output DC power obtained when using an unconventional signal.

\section{A. Intermittent $\mathrm{CW}$}

An early use of a non-conventional waveform to enhance WPT can be found in [9], where the CW signal of a UHF RFID reader was switched ON and OFF with a given duty cycle, $D$ - ratio between ON time (Ton) and cycle period (T). This created a pulsed high PAPR pattern, which enhanced the RF-DC conversion efficiency of the tag's rectifier circuits, and consequently allowed to reduce the average radiated power, while keeping the same communication distance. 
In order to evaluate this effect, a series diode rectifier ${ }^{2}$ (similar to that in Fig.2b) is fed either with a CW signal (with constant amplitude $A$ ) and with an ICW signal (with $\mathrm{ON}$ amplitude equal to $B$ ). Both signals are set to the same average power by doing $B=A / \operatorname{sqrt}(D)$ and the output DC values are compared. As observed in Fig.6, the output voltage produced by a $15 \%$ duty cycle ICW signal (signal with ripple) is higher than that provided by a $\mathrm{CW}$ signal (constant signal) with the same average power. Tables 1 and 2 evaluates the dependency of the output DC voltage on the ICW duty cycle (D), input peak amplitude (B) and PAPR. As can be seen, the lower the duty cycle and the higher the input peak amplitude and PAPR, the higher the output voltage and efficiency gain. On the other hand, the increase in D causes an increase in the output ripple, thus a tradeoff should exist.

It is worth to mention that, if simultaneous power and data transfer is desired (e.g. in passive RFID), then the pulse envelope frequency must be higher than the intended data rate. In this example a $1 \mathrm{GHz}$ signal with $1 \mathrm{MHz}$ square envelope is used.

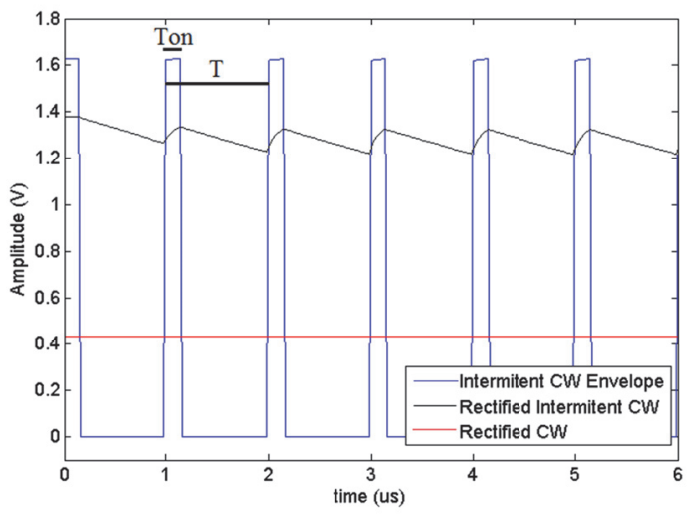

Fig. $6 \mathrm{CW}$ versus intermittent $\mathrm{CW}$ signal, simulated time domain waveform for input ICW envelope (square wave), rectified ICW output

(signal with ripple) and rectified $\mathrm{CW}$ output (constant signal).

Table 1: CW input-output.

\begin{tabular}{|l|l|l|l|l|}
\hline $\begin{array}{l}\text { Input } \\
\text { Amplitude, } \\
\text { A }(\mathrm{mV})\end{array}$ & $\begin{array}{l}\text { Input } \\
\text { PAPR }\end{array}$ & $\begin{array}{l}\text { Input Duty } \\
\text { Cycle, D }\end{array}$ & $\begin{array}{l}\text { Output } \\
\text { DC, V } \\
(\mathrm{mV})\end{array}$ & $\begin{array}{l}\text { Gain } \\
(\mathrm{G} \eta)\end{array}$ \\
\hline 631 & $3 \mathrm{~dB}$ & 1.0 & 434 & $0 \mathrm{~dB}$ \\
\hline
\end{tabular}

Table 2: Intermittent CW input-output as a function of D and PAPR.

\begin{tabular}{|l|l|l|l|l|}
\hline $\begin{array}{l}\text { Duty } \\
\text { cycle, } \\
\text { D }\end{array}$ & $\begin{array}{l}\text { Input } \\
\text { Amplitude } \\
\mathrm{B}=\mathrm{A} / \text { sqrt(D) }\end{array}$ & $\begin{array}{l}\text { Input PAPR } \\
=10 \log (2 / \mathrm{D}) \\
(\mathrm{dB})\end{array}$ & $\begin{array}{l}\text { Output } \\
\mathrm{DC} \\
\left(\mathrm{V}_{\mathrm{DC}}\right)\end{array}$ & $\begin{array}{l}\text { Gain } \\
(\mathrm{G} \eta)\end{array}$ \\
\hline 1.0 & 631 & 3 & 434 & $0 \mathrm{~dB}$ \\
\hline 0.7 & 754 & 4.6 & 538 & 1.87 \\
\hline 0.4 & 997 & 7 & 744 & 2.94 \\
\hline 0.2 & 1408 & 10 & 1096 & 8.04 \\
\hline 0.1 & 1991 & 13 & 1585 & 11.3 \\
\hline
\end{tabular}

\footnotetext{
${ }^{2}$ Passive RFID Tags actually use voltage multipliers, however similar behavior is observed in the single diode rectifier.
}

\section{B. UWB Signals}

Following the same reasoning, Ultra Wide Band (UWB) signals were proposed for efficient low power transmission [10-11]. A train of short duration pulses with relatively high amplitude exhibits a very low average power value (and consequently a high PAPR value). In [10] a conversion efficiency of $50 \%$ was achieved in a Schottky diode voltage doubler fed with a relatively low average power level of $-3 \mathrm{dBm}$.

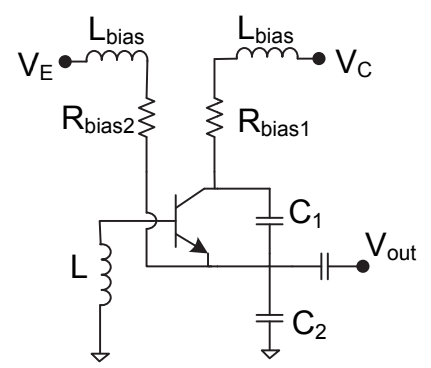

(a)
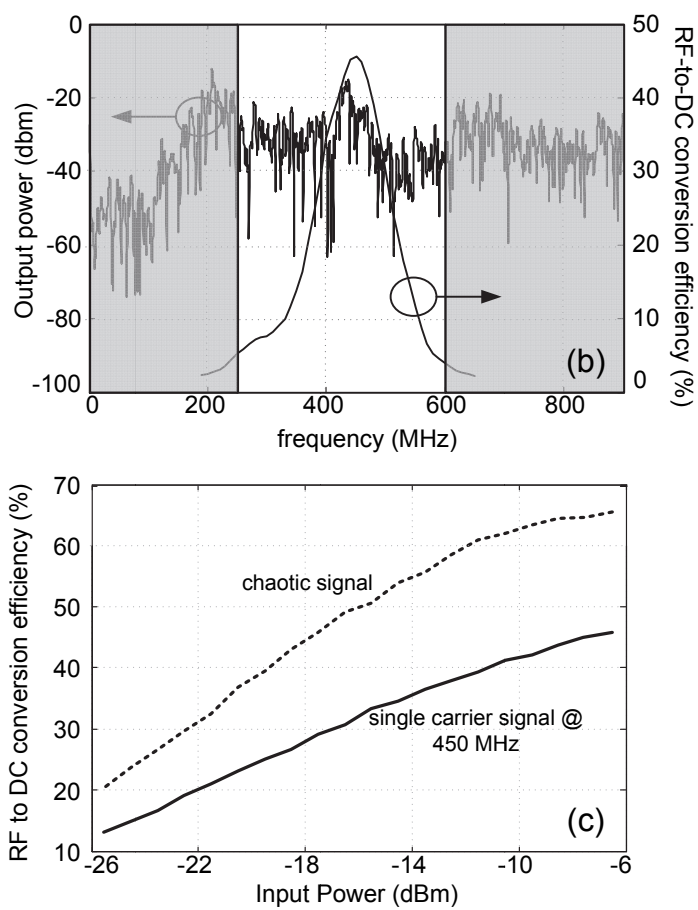

Fig. 7 High PAPR chaotic signals improve RF-DC conversion efficiency in rectifier circuits (a) Chaotic generator (b) Frequency spectrum of chaotic signal and RF-DC conversion efficiency of the rectifier circuit (c) Comparison of RF-DC conversion efficiency when using chaotic signals and single carrier signal.

\section{Chaotic Waveforms}

In [14] the use of a chaotic generator was proposed as a way to create high PAPR signals. A Colpitts based circuit with only one active device (bipolar transistor BFP183W) is used to synthesize a chaotic waveform that has a high PAPR (Fig.7a). As chaotic signals have a continuous and broadband frequency spectrum it is necessary to filter them before their use in WPT systems in order to avoid radiating in restricted frequency bands. 
The chaotic signal used in [14] is centered around $450 \mathrm{MHz}$ and has a PAPR of approximately $6.8 \mathrm{~dB}$ (Fig.7b). The performance of a rectifier circuit in terms of RF-DC conversion efficiency when using this chaotic signal in comparison with a single carrier signal at $450 \mathrm{MHz}$ is performed in [14]. The rectifier circuit is based on the SMS7630 Schottky diode and has an RF-DC conversion efficiency curve centered around $450 \mathrm{MHz}$ (Fig.7b). From Fig. $7 \mathrm{c}$ it can be seen that the use of the chaotic signal leads to an improvement in the RF-DC conversion efficiency of the rectifier circuit of $20 \%$ compared to a single carrier signal with the same average power of $-6.5 \mathrm{dBm}$. As we reduce the input power level the improvement in the RF-DC conversion efficiency is less dramatic but still one can obtain improvements in the order of $15 \%$ for $-20 \mathrm{dBm}$ input power.

\section{Modulated Signals}

In [15] different types of signals including an OFDM and white noise based signal are considered towards maximizing efficiency in WPT systems. Specifically the selected OFDM signal is an LTE FDD downlink OFDM signal with 301 occupied sub-carriers modulated using QPSK, 5MHz bandwidth and a PAPR of $12 \mathrm{~dB}$. The white noise based signal is a band limited signal around $433 \mathrm{MHz}$ and it is synthesized modulating a single carrier by a Gaussian white noise signal provided by an arbitrary waveform generator Agilent 33250A source. The signal has $1 \mathrm{Vpp}$ amplitude and a PAPR of $13.7 \mathrm{~dB}$. In both cases the signals are filtered using a band-pass filter centered around $433 \mathrm{MHz}$ and with a $3 \mathrm{~dB}$ bandwidth of $6 \mathrm{MHz}$.

An experiment is performed in [15] where the performance of a rectifier circuit in terms of RF-DC conversion efficiency is evaluated for the previous two signals and also for a single carrier signal. The carrier frequency for the three signals used in the experiment is $433 \mathrm{MHz}$. Fig. 8a shows the obtained results demonstrating how high PAPR modulated signals can obtain improved performance in rectifier circuits compared to single carrier signals and consequently are suitable for their use in WPT systems. Fig. $8 \mathrm{~b}$ shows the Complementary Cumulative Distribution Function (CCDF) of the PAPR of the envelope of the signals showing that the OFDM signal apart from having a higher maximum PAPR it also has a higher periodicity in the occurrence of the peaks in the signal. This fact leads to even a higher improvement in the RF-DC efficiency if compared with the white noise based signal.
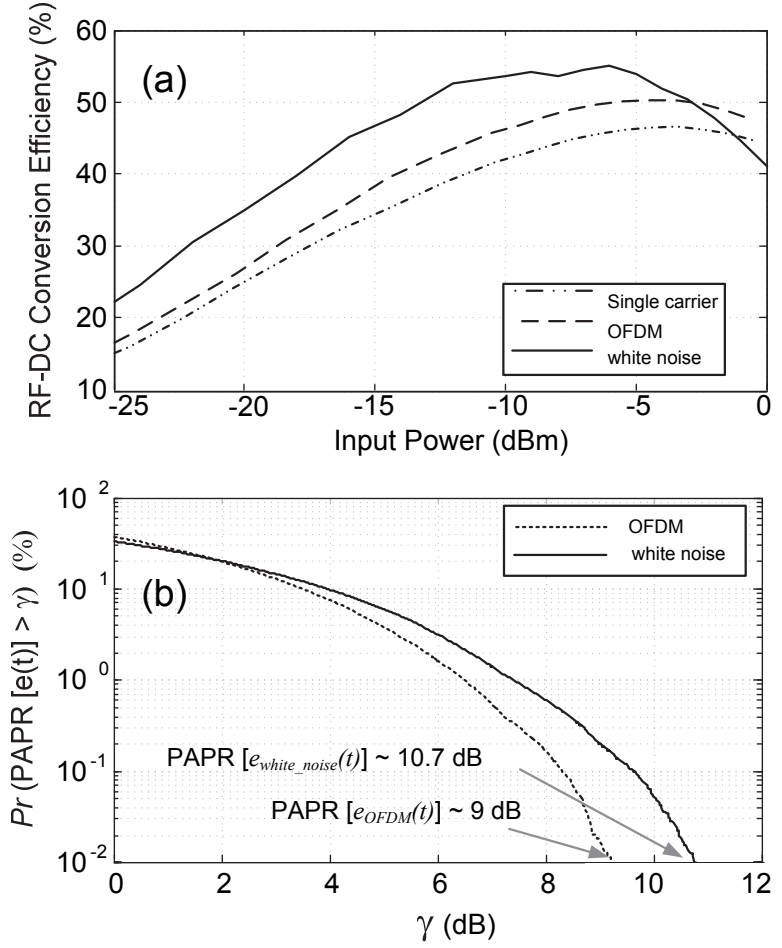

Fig. 8 Evaluation of rectifier performance for different types of signals (a) RF-DC conversion efficiency of a rectifier centered around $433 \mathrm{MHz}$ when using a single carrier, OFDM signal and white noise based signal. (b) CCDF of the PAPR of the selected signals for the experiment.

\section{E. Multi-sines}

Multi-sine signals, commonly used in Orthogonal Frequency Division Multiplex (OFDM) communication systems, have been explored for their use in WPT. In [3-5] the reading range of UHF RFID tags was extended by using a multi-sine scheme. In [7] the non-linear behavior of RFDC converters was investigated and a mathematical model and description were presented to explain the efficiency enhancement in Schottky diode detectors when excited with high PAPR signals. In the experiments conducted in [9] and [10], a multi-sine front-end was integrated in a commercial RFID reader in order to extend its reading range.

\section{Multi-sine design}

A multi-sine signal results from the combination of several sines with different amplitudes, frequencies and phases. The choice of the individual tone characteristics determines the shape of the multi-sine time-domain waveform. As stated before, in order to achieve the maximum PAPR a proper phase arrangement must be selected. Beyond phase optimization, further optimization can be done by playing with the individual tone amplitudes and frequency separation. For instance in [16] Gaussian formats were obtained using a special designed algorithm. 
Fig. 9a) presents a four-tone multi-sine signal, centered ${ }^{3}$ at $100 \mathrm{kHz}$, with a tone spacing of $667 \mathrm{~Hz}$, and with a random phase arrangement. In Fig. 9b) the time domain waveform of a four-tone signal with $0^{\circ}$ phase is depicted, overlapped with a CW with same average power $\left(A_{C W}=\sqrt{N} * A_{\text {Tone }}\right.$, where $\mathrm{A}_{\text {Tone }}$ is the amplitude of the individual tones of (3) and $\mathrm{N}$ is the number of tones, $\mathrm{N}=4$ ). While the random phase signal exhibits low peak values and low PAPR, the $0^{\circ}$ phase signal presents a much higher PAPR than the CW signal, and consequently will provide a higher rectified DC output.

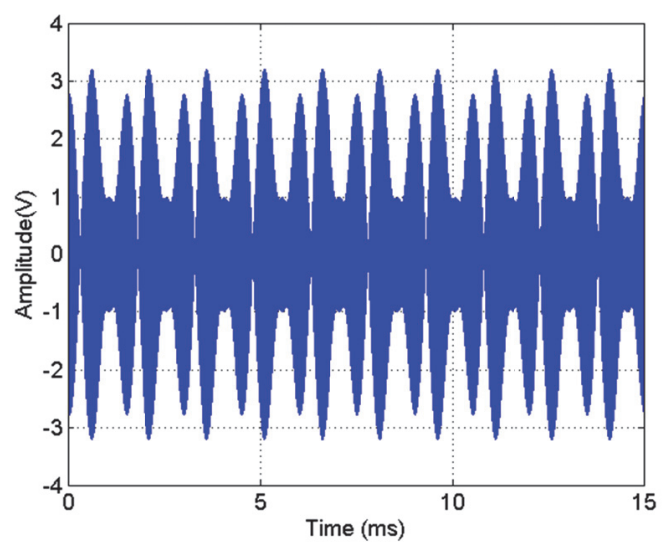

(a)

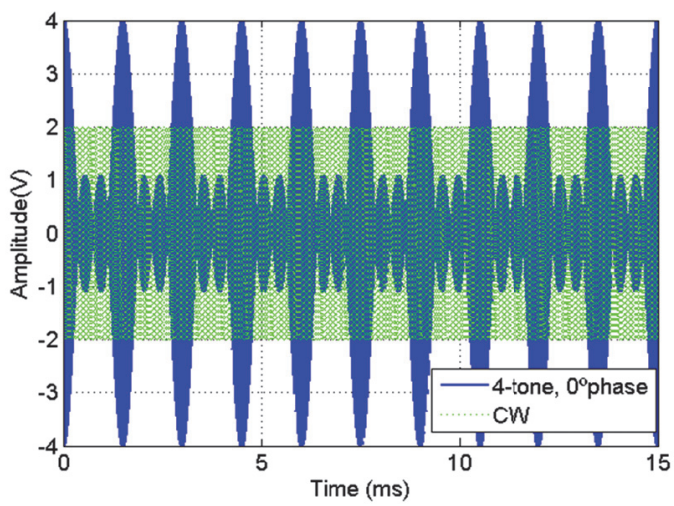

(b)

Fig. 9 Time-domain waveforms, a) four-tone multi-sine with random phase arrangement, c) four-tone with $0^{\circ}$ phase arrangement overlapped with $\mathrm{CW}$ with same average power.

\section{Measured efficiency gain results}

Measurements were conducted to evaluate the efficiency gains obtained with several multi-sine signals. Two RF-DC converter circuits where tested, namely a single diode detector (Fig.10a) [13], commonly used for energy harvesting and WPT, and a 5-stage charge pump or voltage multiplier typically used in passive RFID tags (Fig.10b) [17]. The two circuits operate at $2.3 \mathrm{GHz}$ and $866 \mathrm{MHz}$ band respectively. The RF-DC converters are first fed with a CW signal and afterwards with a multi-sine signal with the same average power as the $\mathrm{CW}$ signal. The converter circuits are tested over a range of input power, and input signal bandwidth and phase arrangement of the multi-sine.

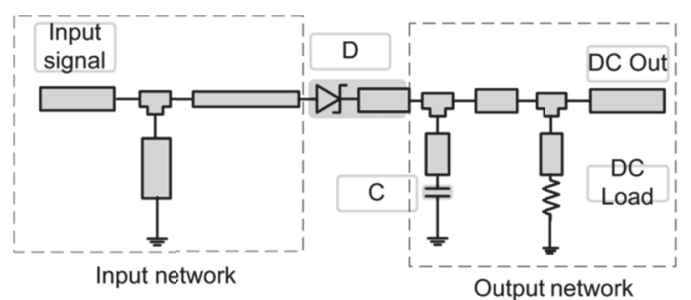

(a)

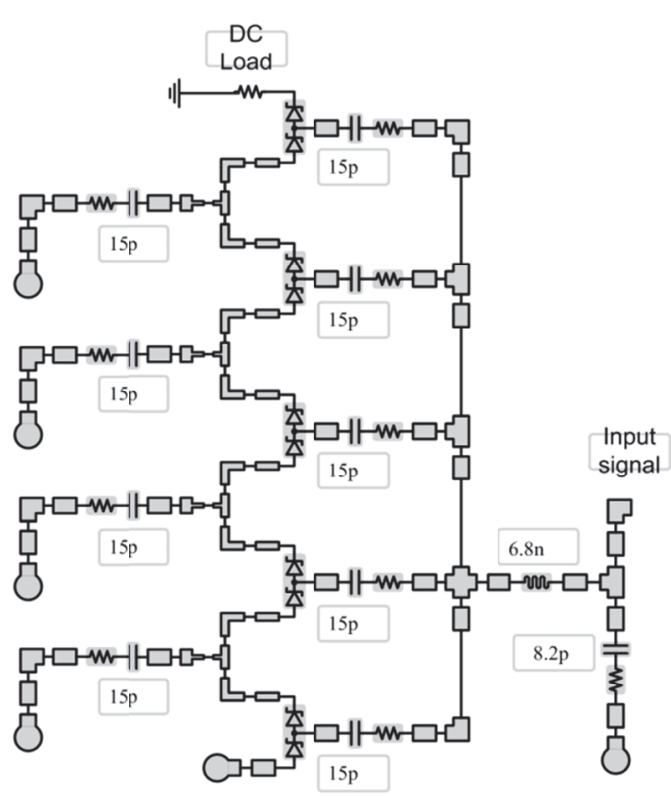

(b)

Fig. 10 Rectifying circuits constructed for tests, a) single diode rectifier operating at $2.3 \mathrm{GHz}$ band, b) charge pump circuit operating at $866 \mathrm{MHz}$.

Figures 11 and 12 provide the DC voltage measurements and the efficiency gain as defined by equation (5). The measurement results support the initial premise that multisine signals provide an efficiency gain over $\mathrm{CW}$ signal. This is true both for the single diode rectifier as well as for the charge pump circuit, and in the second case, it explains the range improvements in passive RFID systems reported in [12] and [19].

\footnotetext{
${ }^{3}$ Center frequency and tone spacing only for illustration purposes.
} 


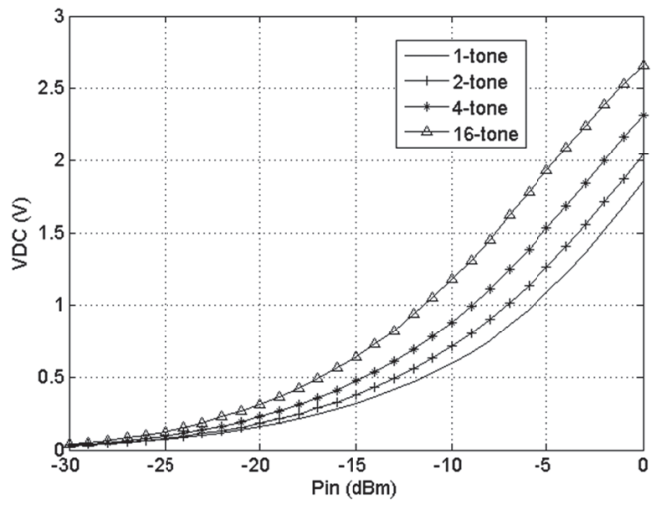

(a)

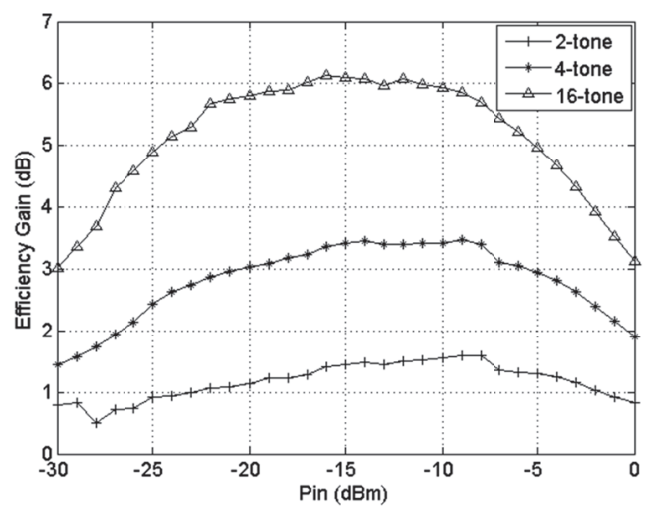

Fig. 71 Single diode rectifier measurements, a) DC voltage as a function of input power, b) efficiency gain as a function of the input power.

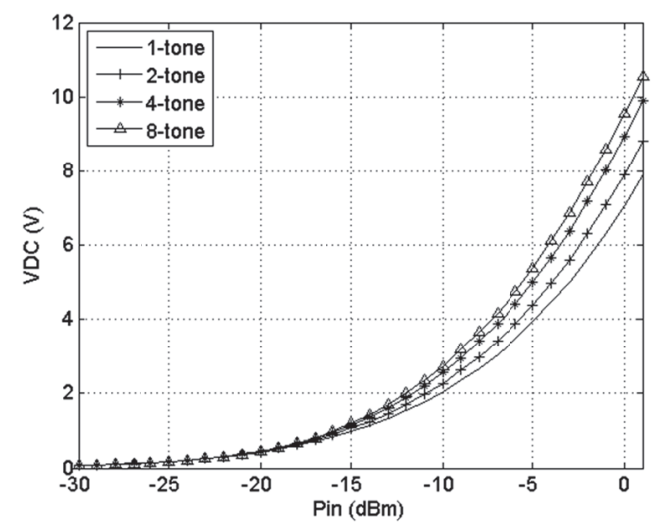

(a)

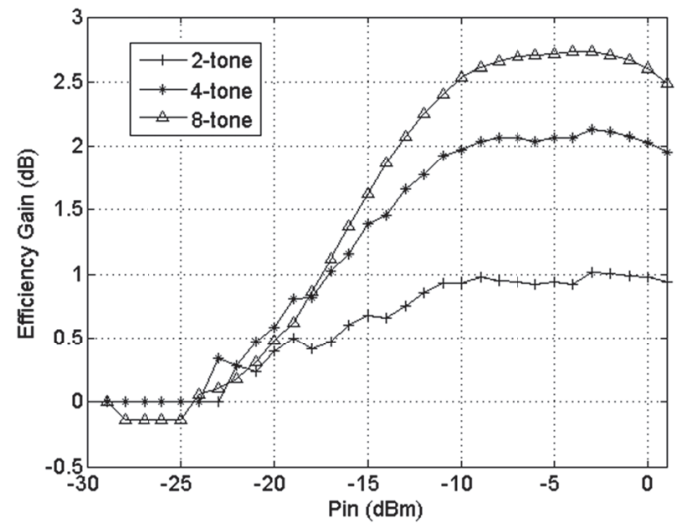

(b)

Fig. 82 Charge pump rectifier measurements, a) DC voltage as a function of input power, b) efficiency gain as a function of the input power.

\section{F. $\quad$ Harmonically spaced Multi-sines}

Multi-sine signals are usually designed to operate on a single band to keep the matching procedure simple at the receiver side. However, to provide a high PAPR signal many subcarriers are needed and thus the frequency spacing between each subcarrier gets significantly lower as the number of subcarriers increases. This kind of signals are characterized by having an envelope that has a peak power period equal to the inverse of the subcarriers spacing frequency. The output low-pass filter needs a high time constant in order to filter out the low intermodulation products that are generated in the rectification process and to avoid a large output ripple that would reduce the DC level generated. Usually, low-pass filters are simple RC circuits. Increasing the capacitor value might not be a good idea since high value capacitor are known to have bad behavior at high frequencies and increasing the value of the load enables us to obtain more DC voltage but reduces the available DC output power. To prevent such phenomena, a multi-band RF-DC converter is considered and tested in this section to obtain a higher frequency spacing between each subcarrier as shown in Fig. 13. If the bands are harmonically spaced, the resulting time domain signal has the same peak voltage of a single band multi-sine. Moreover, it has the same peak voltage frequency of a $\mathrm{CW}$ at the same frequency. Fig. 14 presents such a signal.

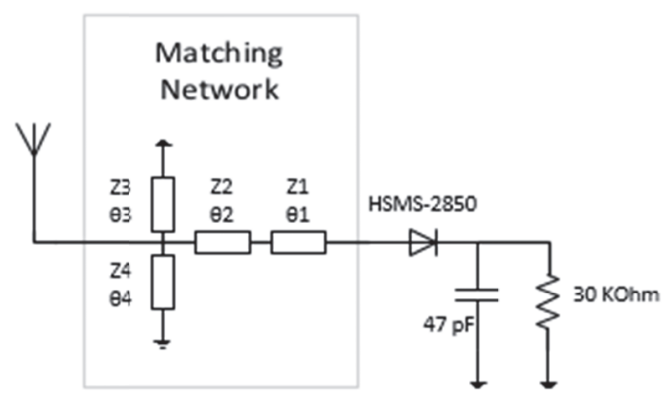

Fig. 93 Proposed dual band RF-DC converter 


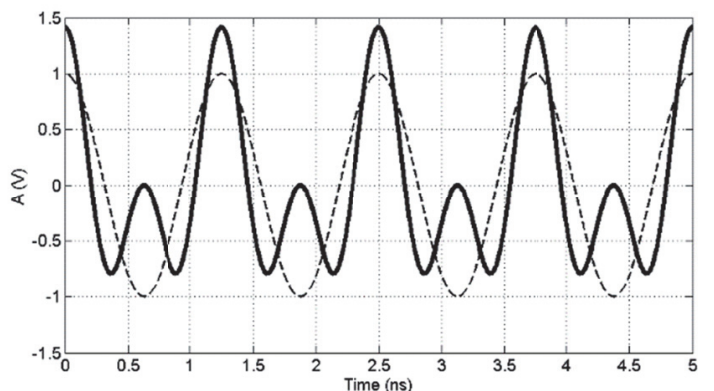

Fig. 104 Time domain waveforms of a CW at $900 \mathrm{MHz}$ and an harmonically spaced multi-sine, 2 subcarriers at 900 and $1800 \mathrm{MHz}$ with same total power

The most challenging issue of using these signals is the matching procedure at the receiver. For two subcarriers, similar to the single-stub matching network at a single frequency, two section of transmission lines are connected in series with the circuit, and transforms its input impedances to normalized unit conductance at the two designated frequencies simultaneously. The resulting susceptances are then cancelled out by a two shunt stub at the two frequencies, as shown in Fig. 13 [22]. The circuit was then optimized for the two bands and for $-15 \mathrm{dBm}$ of total input power (Fig. 15).

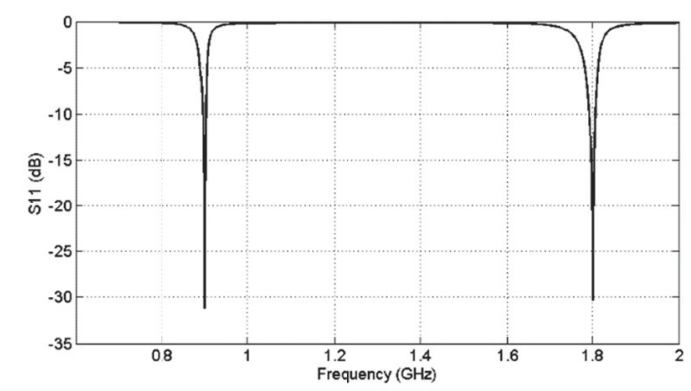

Fig. 115 Simulated S11 of the dual band RF-DC converter with $-15 \mathrm{dBm}$ of input power.

Fig. 16 shows the simulated results when excited with different signals. It can be observed that, for this circuit, the harmonic spaced multi-sine provides the best results in terms of DC output voltage and power conversion efficiency considering all excitation signals with same power. This is due to the better synergy between the output filter time constant and input peak voltage period. The 30 $\mathrm{k} \Omega$ load was chosen to maximize both voltage gain and power conversion efficiency.

It is possible to observe that this kind of signal enables the rectifier to generate more DC voltage at higher input powers, virtualizing a larger diode breakdown voltage. In fact, the resulting time domain waveform of the harmonic spaced multi-sine is asymmetric, providing high positive peak voltage and lower negative peaks, as the number of harmonics increases. If we look at the receiver, subcarriers must arrive at the diode's input at $0^{\circ}$ phase arrangement in order to show a high PAPR. As it is, the phase synchronism at the transmitter must be calibrated based on the amount of phase shift that each subcarrier suffers on the matching network, as they are quite separated in frequency. On this simulation, a phase shift of $200^{\circ}$ was needed between carriers at source to provide the maximum PAPR at the diode's input. More work and progress is being done about this approach and more results are expected in the near future.

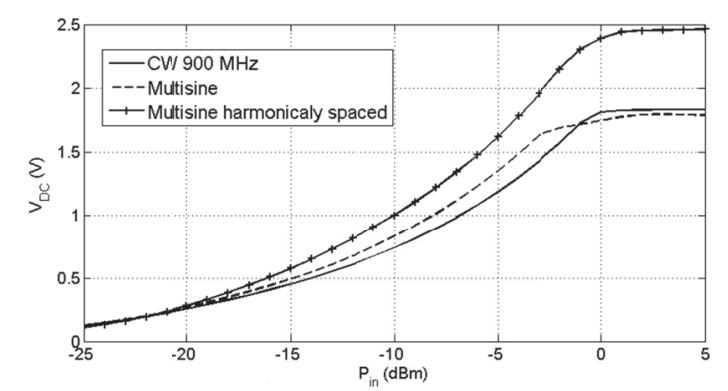

(a)

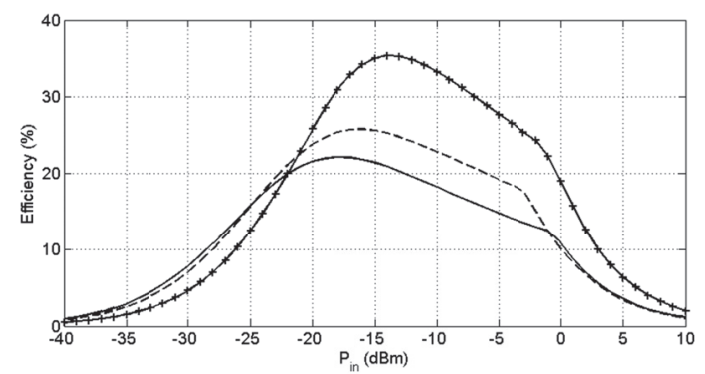

(b)

Fig. 126 Simulated results when excited with a $900 \mathrm{MHz} \mathrm{CW}, 899+901$ $\mathrm{MHz}$ multi-sine and a $900+1800 \mathrm{MHz}$ multi-sine. a) Output DC voltage generated b) Power conversion efficiency achieved.

\section{DISCUSSION: Pros AND CONS OF EACH APPROACH}

The approaches presented in this paper present a tradeoff between efficiency and spectrum occupancy, system complexity and generation approaches.

Intermittent $\boldsymbol{C W}$ : The disadvantage of this solution is the spectral inefficiency caused by spectrum re-growth (due to the intermittent regime). At least, some low-pass filtering should be applied to the intermittent pattern before transmitting the signal, which can reduce the predicted PAPR.

$\boldsymbol{U W B}$ : The main drawback of the UWB approach is the increased spectral bandwidth and the need for wideband antennas and components. Furthermore, contrary to multisine signals that can be incorporated conventional RFID systems (as was done in [12] and [18-19]), the UWB scheme cannot be directly applied to these systems, which are narrowband. Ultra-wide band circuits are needed.

Chaotic signals: As a disadvantage they have a continuous and broadband spectrum so it is necessary to filter them in order to avoid radiating in restricted or not allowed 
frequency bands. On the other hand synthesizing chaotic waveforms can be less complex than synthesizing other types of signals; as an example the chaotic generator of [14] where a single active device circuit suffices.

Multi-sines: it is necessary to generate multi-carrier components, which increases the complexity of the transmitter (unless envelope amplification techniques such as ET - envelope tracking is used). rectifier must accommodate the multi-sine signal bandwidth. A generation alternative was presented in [20-21] that can overcome some of these challenges by space power combining sines in the air interface.

In all cases the amplification of high PAPR signals is challenging which requires efficient approaches to amplify and transmit such high PAPR signals. For instance, in [21], two architectures were proposed to efficiently create high PAPR multi-sines. The first one consists of individually transmitting single tone signals that are externally locked to a common reference signal to establish a phase reference. The second one is based on mode-locked oscillator schemes, where no external reference is required, but advantage is taken of the synchronization phenomena to establish the phase reference.

Harmonic signals: need for multi-band circuit design at the receiver side and requires the generation of harmonic multifrequency signals at the transmitter. The rectifier device is required to operate in the selected frequency harmonics. Nevertheless preliminary results show that these are probably the signals that achieve better results in conventional RF-DC converters.

\section{REFERENCES}

[1] Anton A. Huurdeman, The worldwide history of Telecommunications, John Wiley \& Sons, Inc., Hoboken, New Jersey, 2003.

[2] N. Tesla, "The Transmission of Electric Energy Without Wires" (The Thirteenth Anniversary Number of the Electrical World and Engineer). New York: McGraw-Hill, Mar. 5, 1904.

[3] Biography of Ernst F. W. Alexanderson, IEEE Global History Network.

http://www.ieeeghn.org/wiki/index.php/Ernst F. W. Alexanderson

[4] W. C. Brown, "Electronic and mechanical improvement of the receiving terminal of a freespace microwave power transmission system", Raytheon Company, Wayland, MA, Tech. Rep. PT-4964, NASA Rep. CR-135194, Aug. 1977.

[5] A. Georgiadis, G. Andia and A. Collado, "Rectenna Design and Optimization Using Reciprocity Theory and Harmonic Balance Analysis for Electromagnetic (EM) Energy Harvesting”, IEEE Antennas and Wireless Propagation Letters, Vol.9, 2010.

[6] Zied Harouni, Lotfi Osman and Ali Gharsallah, "Efficient $2.45 \mathrm{GHz}$ Rectenna Design with High Harmonic Rejection for Wireless Power
Transmission", IJCSI International Journal of Computer Science Issues, Vol. 7, Issue 5, September 2010

[7] Ken Hatano, Naoki Shinohara, Tomohiko Mitani, Kenjiro Nishikawa, Tomohiro Seki, Ken Hiraga, "Development of Class-F Load Rectennas", International Microwave Workshop Series on Innovative Wireless Power Transmission: Technologies, Systems, and Applications (IMWS), 2011

[8] Scheeler, R. Korhummel, S. Popovic, Z. "DaulFrequency Ultralow-Power Efficient 0.5g Rectenna”, IEEE Microwave Magazine, Vol. 15, Issue 1. 2014

[9] H. Matsumoto, K. Takei, "An Experimental Study of Passive UHF RFID System with Longer Communication Range", Proceedings of Asia-Pacific Microwave Conference 2007, pp. 1-4.

[10] Chun-Chih Lo, Yu-Lin Yang, Chi-Lin Tsai, Chieh-Sen Lee, and Chin-Lung, Yang, "Novel Wireless Impulsive Power Transmission to Enhace the Convertion Efficiency for Low Input Power" , Microwave Workshop Series on Innovative Wireless Power Tansmission, 2011

[11] Yu-Lin Yang, Chin-Lung Yang, Chi-Lin Tsai, and Chieh-Sen Lee, "Efficiency Improvement of the Impulsive Wireless Power Transmission”, Microwave Workshop Series on Innovative Wireless Power Tansmission, 2011

[12] M. S. Trotter, J. D. Griffin and G. D. Durgin "PowerOptimized Waveforms for Improving the Range and Reliability of RFID Systems", IEEE International Conference on RFID, 2009.

[13] A. Soares Boaventura, N.B. Carvalho "Maximizing $D C$ Power in Energy Harvesting Circuits Using Multisine Excitation", IMS2011 - International Microwave Symposium, Baltimore, USA, June, 2011.

[14] A. Collado, A. Georgiadis, "Improving Wireless Power Transmission Efficiency Using Chaotic Waveforms," in Proc. IEEE MTT-S IMS 2012, Montreal, 17-22 June 2012

[15] A. Collado, A. Georgiadis, "Optimal Waveforms for Efficient Wireless Power Transmission," Microwave and Wireless Components Letters, IEEE, vol.24, no.5, pp.354,356, May 2014.

[16] Carvalho, N.B.; Remley, K.A.; Schreurs, D.; Card, K.C., "Multisine signals for wireless system test and design" Microwave Magazine, IEEE , vol.9, no.3, pp.122,138, June 2008

[17] Fernandes, R.D.; Carvalho, N.B.; Matos, J.N., "Design of a battery-free wireless sensor node," EUROCON International Conference on Computer as a Tool (EUROCON), 2011 IEEE , vol., no., pp.1,4, 27-29 April 2011

[18] C.R.Valenta, G.D. Durgin, "Rectenna performance under power-optimized waveform excitation," RFID (RFID), 2013 IEEE International Conference on , vol., no., pp.237,244, April 30 2013-May 2013.

[19] A. Soares Boaventura, N.B. Carvalho, "Extending Reading Range of Commercial RFID Readers”, IEEE 
Transactions on Microwave Theory and Techniques, vol.61, no.1, pp.633,640, Jan. 2013.

[20] R. A. York, R. Compton, "Coupled-Oscillator Arrays for Millimeter-Wave Power-Combining and ModeLocking”, IEEE International Microwave Symposium, California USA, 1992.

[21] A. Soares Boaventura, A. Collado, A. Georgiadis and N.B. Carvalho, "Spatial Power Combining of Multisine Signals for Wireless Power Transmission Applications", IEEE Transactions on Microwave Theory and Techniques, 2014, 